Check for updates

Cite this: RSC Adv., 2019, 9, 20295

\title{
Light induced reversible structuring of photosensitive polymer films $\dagger$
}

\author{
Joachim Jelken and Svetlana Santer (D) *
}

In this paper we report on photoswitchable polymer surfaces with dynamically and reversibly fluctuating topographies. It is well known that when azobenzene containing polymer films are irradiated with optical interference patterns the film topography changes to form a surface relief grating. In the simplest case, the film shape mimics the intensity distribution and deforms into a wave like, sinusoidal manner with amplitude that may be as large as the film thickness. This process takes place in the glassy state without photo-induced softening. Here we report on an intriguing discovery regarding the formation of reliefs under special illumination conditions. We have developed a novel setup combining the optical part for creating interference patterns, an AFM for in situ acquisition of topography changes and diffraction efficiency signal measurements. In this way we demonstrate that these gratings can be "set in motion" like water waves or dunes in the desert. We achieve this by applying repetitive polarization changes to the incoming interference pattern. Such light responsive surfaces represent the prerequisite for providing practical applications ranging from conveyer or transport systems for adsorbed liquid objects and colloidal particles to generation of adaptive and dynamic optical devices.

Received 5th April 2019

Accepted 12th June 2019

DOI: 10.1039/c9ra02571e

rsc.li/rsc-advances temporally without undesired contact with electrodes, wires or heating elements. ${ }^{19-23}$ One of the strategies to render a polymer photo-responsive is to modify it with photo-reactive groups among which azobenzenes are the most common. Azobenzene containing polymers belong to a class of functional materials exhibiting a strong mechanical response upon applying external radiation fields. Although the radiation only acts on the photosensitive azobenzene moieties by inducing trans-cis isomerization and thus configurational changes, as side groups in a polymer chains they can affect the system as a whole. Such materials have extensively been studied and were proposed for the construction of holographic gratings and optical data storage devices. ${ }^{\mathbf{1 0}}$ Very peculiar phenomena are obtained when azobenzene modified physisorbed polymer films or the even more exotic polymer brushes are exposed to irradiation exhibiting interference patterns (IP). ${ }^{24-32}$ Following the IP's periodicity either with respect to intensity or polarization distribution, the polymer topography is modulated into a wave like form with period equal to optical one. ${ }^{33-36}$ The mechanism of SRG formation is related to the generation of strong, internal optomechanical stresses. Since the polymer material is in a glassy state, the opto-mechanical stresses needed for the deformation of mechanically stable polymer films (Young modulus of several GPa) must be quite high of $100 \mathrm{MPa}$ up to $\sim 1 \mathrm{GPa}$, as was recently shown. ${ }^{37-40}$ The process can be viewed as follows: under irradiation inducing cyclic trans-cis-trans isomerization, ${ }^{\mathbf{4 1}}$ the azobenzene molecules rotate and re-orient perpendicularly to the electrical field vector (formation of bulk birefringence grating) causing a re-orientation of the polymer backbones to
Institute of Physics and Astronomy, University of Potsdam, 14476 Potsdam, Germany. E-mail: santer@uni-potsdam.de

$\dagger$ Electronic supplementary information (ESI) available. See DOI: 10.1039/c9ra02571e 
which they are attached. This creates local gradients in internal stresses within the polymer material, varying with the distribution of the interference pattern and thus induces a viscoplastic deformations of the solid material (formation of topographical grating). ${ }^{\mathbf{4 2}-47}$ In fact, the inscribed SRG topography is stable over years at room temperature and can be erased only by further intense treatment such as either irradiation with a circularly polarized beam ${ }^{48}$ or by heating ${ }^{49}$ the polymer sample above the glass transition temperature.

Usually the dynamics of the SRG formation is probed by measuring the changing diffraction efficiency of the formed grating. Here a probe beam not affecting the azobenzene moieties is focused on the relief structure and the change in intensity of the first order diffraction peak is recorded as a function of time. The surface grating and the birefringent grating contribute to the signal and it is hard to separate these two components. ${ }^{50-52}$ Another approach is to directly measure the change in the SRG using an atomic force microscope (AFM). ${ }^{39,53-55}$ The drawback of the latter methodology is that there will be no information obtained about the orientation process in the bulk polymer. Here we present a novel set-up which combines these two approaches.

An AFM is integrated into a two beam interference set-up generating the interference pattern. This allows the in situ acquisition of surface topography changes. At the same time the diffraction efficiency (DE) signal is measured to obtain information about the bulk birefringence. Integrating a delay stage into the two beam setup allows controllable spatio-temporal shifts of the interference pattern, which locally appears as a redistribution of the electrical field vector. In this way gradual shifts or even more dynamic fluctuations in the polymer topography and the bulk birefringence can be generated, for instance, mimicking a passing wave train the speed of which can be adjusted. One may, for instance, stop migration at some point and resume at a desired later time just by switching light on and off. Additionally, we introduce a fast method of optically erasing SRGs by manipulating the illumination pattern in a particular way. One may either completely erase the topography and bulk birefringence grating or erase the primary topography grating. The time needed for erasure (only a few seconds) is much shorter than that needed for single beam irradiation (several minutes to hours) and heating (several tens of seconds during direct heating by laser irradiation ${ }^{49}$ to several minutes and hours under external temperature increase) to achieve a comparably flattening.

\section{Experimental part}

\section{Materials and methods}

Photosensitive polymers. Poly[1-[4-(3-carboxy-4-hydroxyphenylazo)benzenesulfonamido]-1,2-ethanediyl, sodium salt] (Pazo) and poly[(methyl methacrylate)-co-(disperse red 1 acrylate)] (poly(MMA-co-DR1A)) were purchased from SigmaAldrich. The chemical structures of Pazo and poly(MMA-coDR1A) are shown in the inset of Fig. 2a and b. The Pazo polymer solution was prepared by dissolving $170 \mathrm{mg}$ Pazo in $1 \mathrm{ml}$ solution containing a mixture of 95\% methoxyethanol and 5\% ethylene glycol. The poly(MMA-co-DR1A) polymer was dissolved in chloroform to achieve a concentration of $60 \mathrm{mg} \mathrm{ml}^{-1}$. The polymers were chosen to represent two extrema, i.e. Pazopolymer does not have $T_{\mathrm{g}}$ and cannot be flattened by arising temperature (as discussed in the paper), poly(MMA-co-DR1A) has $T_{\mathrm{g}}$ around $100{ }^{\circ} \mathrm{C}$, and can be flattened thermally.

Sample preparation. The polymer films were prepared by spin casting $100 \mu \mathrm{l}$ of the polymer solution on thin glass slides at $3000 \mathrm{rpm}$ for one minute. This results in a film thickness of 1 $\mu \mathrm{m}$ in the case of the Pazo polymer and of $600 \mathrm{~nm}$ for the pol$\mathrm{y}$ (MMA-co-DR1A). The film thickness was directly obtained with the help of an atomic force microscope and the cross-section analysis of a scratch within the polymer film.

\section{Methods}

The interference pattern is generated using a homemade twobeam interferometer with a continuous wave diode pumped solid state laser of the wavelength $491 \mathrm{~nm}$ (Cobolt Calypso). The beam diameter is set to $4 \mathrm{~mm}$ and the total intensity to $200 \mathrm{~mW}$ $\mathrm{cm}^{-2}$. To probe the diffraction efficiency a $633 \mathrm{~nm}$ HeNe-Laser (Uniphase) with a beam diameter of $3 \mathrm{~mm}$ and an intensity of 30 $\mathrm{mW} \mathrm{cm} \mathrm{cm}^{-2}$ is focused on the sample.

The in situ atomic force microscope (AFM) measurements are performed using a PicoScan (Molecular Imaging) AFM working in intermittent contact mode. The scan-speed of the AFM is set to $1 \mathrm{~Hz}$ with a scan-area of $10 \times 10 \mu \mathrm{m}$ and a resolution of $512 \times 512$ pixel. Commercial tips (Nanoworld-Point probe) with a resonance frequency of $130 \mathrm{kHz}$, and a spring constant of $15 \mathrm{~N} \mathrm{~m}^{-1}$ are used for measurements.

Commercially available Si-Detectors (Thorlabs DET 100A/M) are used in the diffraction efficiency (DE) set-up to measure the intensity of the diffracted probe beam. A longpass filter (600 $\mathrm{nm}$ ) was placed in front of each photodiode in order to be only sensitive to the probe beam. The set-up is controlled and the signals are recorded by an $\mathrm{AD} / \mathrm{DA}$ converter (Kolter Electronic, PCI-AD12N-DAC2). The software Profilab-Expert (Abacom) for visual programming is used to synchronize the DE and twobeam-interference set-up. The intensity of the diffracted light is recorded every $200 \mathrm{~ms}$.

In order to change the position of one mirror in the twobeam-interference set-up to introduce a phase delay between the two interfering beams, a piezo stack actuator (PiezoSystemJena, PA8-14 SG) with a closed loop feedback system and a travel maximum of $9.5 \mu \mathrm{m}$, controlled by a piezo-controller (Piezo System Jena, 12V40SG), is used. Additionally a Pockels cell (Thorlabs, EO-PC-550) acting as an optical switch is integrated into the system for fast switching between the two-beaminterference lithography and single beam erasure experiment. The voltage is supplied by a high voltage amplifier (Trek 610D) which also allows driving the Pockels cell as a lambda quarter or lambda half wave plate depending on the applied voltage.

The AFM measurements performed ex situ (measurements of film thickness and SRG height before and after thermal treatment) are carried out using an NTEGRA (NT-MDT) AFM operating in intermittent contact mode. Commercial tips (Nanoworld-Point probe) with a resonance frequency of 320 
$\mathrm{kHz}$, and a spring constant of $42 \mathrm{~N} \mathrm{~m}^{-1}$ are used for these measurements. All experiments are carried out under yellow light in the laboratory (to avoid undesirable photoisomerization) and under ambient conditions, i.e., at room temperature with a relative humidity of $55 \%$. The whole set-up (see Fig. 1) was covered with a non-transparent encapsulation in order to avoid any influence of the environment on the measurement (room light, air circulation).

\section{Results and discussion}

In order to study the surface relief grating (SRG) formation a novel set-up consisting of three parts is designed enabling in situ recording the change of the SRG-height and diffraction efficiency (DE) as a function of irradiation time (Fig. 1). The first part contains a two beam interference set-up that permits to generate well-defined spatiotemporal intensity or polarization interference patterns by changing polarization of two interfering beams in a controlled manner. In this part of the set-up the laser beam $(\lambda=491 \mathrm{~nm})$ is spatially expanded and then collimated with a pair of focusing and collimating lenses and a pinhole (Fig. 1). Additionally, a $50: 50$ beam splitter is added in order to separate the initially single beam into two beams of the same intensity. These two beams then pass through a set of wave plates and polarizers allowing independent control of intensity and polarization. For instance, adding a lambda quarter plate to each of the beam paths of the interference setup, one with an angle of $+45^{\circ}$ and the second with an angle of $-45^{\circ}$ with respect to the optical axis, results in the right-left- circular interference pattern (RL). The second part of the home made set-up is an atomic force microscope (AFM) enabling measurements of the polymer topography changes in situ, i.e. even while irradiation conditions are varying. The sample is orientated with the polymer surface pointing towards the AFM tip, such that irradiation is "from below", i.e., through the glass surface (Fig. 1).

To obtain at the same time information about the alignment of the azobenzene side chains in the polymer film, a red probe laser beam (HeNe, $633 \mathrm{~nm}$ ) is integrated into the set-up. Its wave length of $633 \mathrm{~nm}$ falls out of the absorption bands of both polymers studied in this work and does not affect the polymer film, so that diffraction efficiency (DE) of the formed SRG can be recorded simultaneously. With our set-up we can acquire the intensity of the diffraction pattern in reflection mode in order to determine the DE of the first order diffraction (Fig. 1). To calibrate the DE, a beam splitter with the ratio of (T90/R10) is used in the DE set-up, such that $90 \%$ of the light arrives on the sample and $10 \%$ on a photodiode. The signal of this photodiode is recorded during the whole measurement for controlling the stability of the probe beam (and its intensity $I_{0}$ prior to arrival at the sample) during the experiment. The diffraction efficiency is defined as the ratio of the intensity of diffraction order $\left(I_{\mathrm{DO}}\right)$ and the intensity of the incoming light $\left(I_{0}\right): \eta=\frac{I_{\mathrm{DO}}}{I_{0}}$, where $I_{0}$ is $90 \%$ of the total intensity of the probe beam. The DE set-up additionally includes a lambda quarter wave plate converting the polarization of the probe beam from linear to circular. By adding a polarizer one can deliberately set the polarization state of the probe beam (e.g. S- or P-polarization). By adding

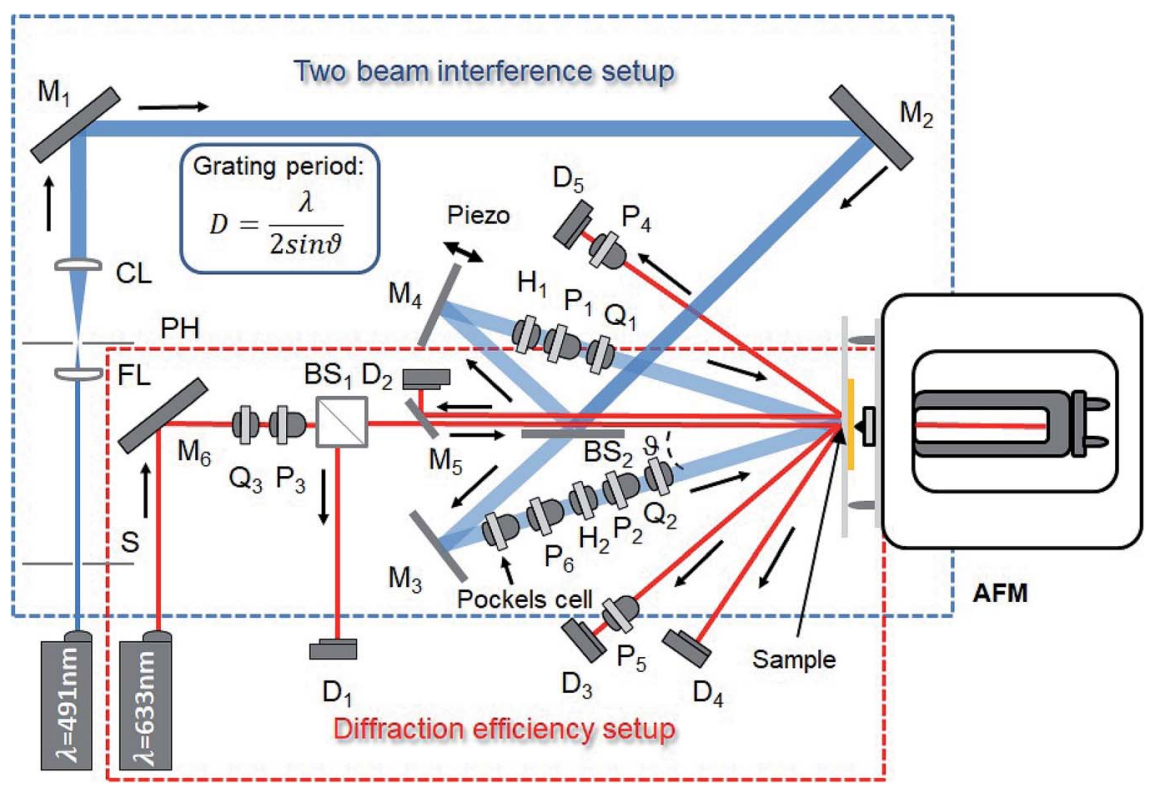

Fig. 1 Sketch of the experimental set-up consisting of three parts: (i) a two beam interference part for generation of the interference pattern (blue laser line), (ii) an atomic force microscope (AFM) for in situ (during irradiation) recording of the surface morphology and (iii) a diffraction efficiency (DE) set-up (red laser line) enabling the collection of information about the birefringence grating in situ. The mirror $\mathrm{M}_{4}$ is combined with a piezo actuator to shift the position of the mirror and thereby to introduce a phase delay between the two interfering beams resulting in a lateral shift of the whole interference pattern along the polymer film. In order to erase the SRG by irradiation, a Pockels cell is placed in one of the two interfering beam lines $(S=$ shutter, $M=$ mirror, $D=$ detector, $P=$ polarizer, $H=$ half-wave-plate, $B S=$ beam $s p l i t t e r, Q=$ quarter-waveplate, $\mathrm{CL}=$ collimating lens, $\mathrm{FL}=$ focusing lens, $\mathrm{PH}=$ pin hole). 
a polarizer in front of the photodetector, one can measure both components of the diffracted light: polarized in plane (further denote as $1^{\text {st }}$ order DE (P), measured by photodiode $\mathrm{D}_{3}$ ) and out of plane (cross-polarized, further denote as $1^{\text {st }}$ order DE (S), measured by photodiode $\mathrm{D}_{5}$ ) with the probe beam. From the cross-polarized component one can directly obtain information about the birefringent grating, i.e. alignment of the azobenzene side chains within the polymer film. The set-up allows also to record the $2^{\text {nd }}$ order $\mathrm{DE}$ (photodiode $\mathrm{D}_{4}$ ) and the directly reflected light $\left(0^{\text {th }}\right.$ order of diffraction, photodiode $\left.\mathrm{D}_{2}\right)$. Adding a piezo actuator to the set up permits nanometer sized position control of mirror $\mathrm{M}_{4}$ (see Fig. 1) for shifting the interference pattern across the polymer film plane in a defined way. Repositioning the mirror simply introduces a phase delay between the two interfering beams, resulting in a change of the local distribution of the polarization vector within interference pattern. A Pockels cell and a polarizer are further placed in the second beam line acting as a fast optical switch. Applying the "lambda half" voltage periodically to the Pockels cell one can rotate the polarization of the writing beam by $90^{\circ}$ such that the light either passes the polarizer or is blocked. In this way, we are able to switch rapidly between two-beam interference and the single beam "erase" configuration of the set-up. The three different set-ups: two beam interference, AFM and DE acquisition are controlled and operated with software specifically designed by us to record signals of the photodiodes, control the irradiation shutter, position the mirror and apply voltage on the Pockels cell. The computer generated signals regulate, with the help of an $\mathrm{AD} / \mathrm{DA}$ converter, the irradiation, the voltage send to the piezo-controller and the high-voltage-amplifier used for driving the Pockels cell. These signals are recorded in the diffraction efficiency set-up as well as with the AFM by sending the signals to the aux-input of the AFM controller.

Fig. 2 shows the in situ recorded SRG height and DE signal for the Pazo and poly(MMA-co-DR1A) polymer films as a function of irradiation time. The probe beam is p-polarized for all results discussed in this paper, while one photodiode measures the intensity of the first order diffraction in-plane $\left(1^{\text {st }}\right.$ order DE
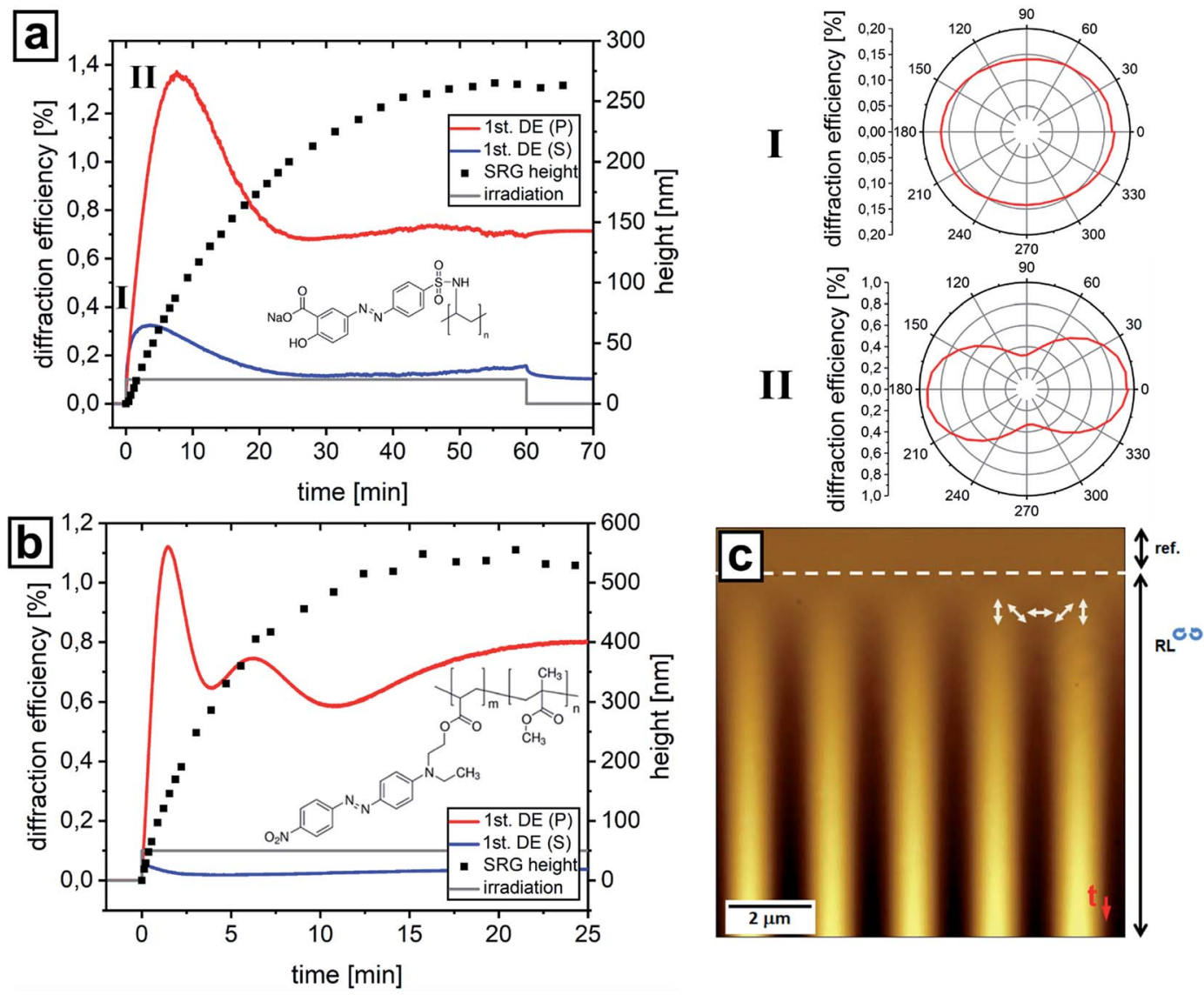

Fig. 2 (a) In situ recorded SRG height and diffraction efficiency of the $1^{\text {st }}$ order of the Pazo polymer film $\left(h_{\text {Pazo }}=1 \mu \mathrm{m}\right)$ irradiated with RL interference pattern as a function of time. The polarization analysis of the $1^{\text {st }}$ order DE signal is shown for two different irradiation times $(I=30 \mathrm{~s}$, II $=3 \mathrm{~min}$ ) on the right. At the beginning the diffracted light is circularly polarized, but with time the component pointing along the polarization of the probe beam dominates. (b) In situ recorded SRG height and $1^{\text {st }}$ order DE of the poly(MMA-CO-DR1A) polymer film $\left(h_{\text {poly }(M M A-c o-D R 1 A)}=600\right.$ $\mathrm{nm}$ ) irradiated with RL IP as a function of irradiation time. (c) AFM micrograph of the Pazo polymer film during irradiation, the direction of scanning is from top to bottom documenting the temporal evolution of polymer topography (the direction of scanning is indicated by the red arrow at the bottom right corner) as a function of irradiation time. The AFM scanning starts without irradiation (flat topography), at the position

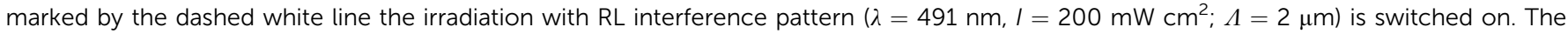
distribution of the electric field vector relative to the topography maxima and minima is shown by white arrows. 
(P)), and the second one measures first order diffraction out-ofplane $\left(1^{\text {st }}\right.$ order DE (S)). The presence of the out-of-plane component of the signal indicates that due to the interaction with the sample the polarization of the p-probe beam is rotated. In the case of the Pazo polymer, the DE signal of the first order diffraction on both photodiodes (red and blue curves in Fig. 2a) continuously increases during irradiation with a RL interference pattern (IP). The DE signals in-plane (red curve, $1^{\text {st }} \mathrm{DE}(\mathrm{P})$ ) and out of plane (blue curve, $1^{\text {st }} \mathrm{DE}(\mathrm{S})$ ) start to decrease when the SRG height reaches $120 \mathrm{~nm}$ and $60 \mathrm{~nm}$, respectively. This behavior follows directly from the Raman-Nath theory ${ }^{56,57}$ and gives rise to describe intensity variation as a function of SRG height by Bessel function first kind of order. The ratio of the inplane and out-of-plane component of the $1^{\text {st }}$ order DE is changing with time. At the beginning (after $30 \mathrm{~s}$ of irradiation) the first order diffraction is circularly polarized (Fig. 2a(I)), while for longer irradiation times (more than $3 \mathrm{~min}$ ) the inplane component is dominating (Fig. 2a(II)). The polar plot in Fig. 2a(I and II) is measured by switching off the pump beam and recording the intensity of the first order diffraction as a function of rotation angle by rotating the polarizer in front of photodiode $\mathrm{D}_{3}$ (in plane component). After 1 hour irradiation the SRG height is $260 \mathrm{~nm}$. The saturation in the SRG growth under irradiation with RL interference patterns for a $1 \mu \mathrm{m}$ thick film is achieved after $c a$. 10 hours of irradiation at which the height is around $900 \mathrm{~nm}$.

In the case of the second polymer studied here (poly(MMAco-DR1A)), the saturation of the SRG height is already achieved after 15 minutes of irradiation with the final value of $550 \mathrm{~nm}$ (total film thickness of $600 \mathrm{~nm}$ ). The DE is showing similar behavior as in the case of the Pazo polymer film, but due to the rapid evolution of the grating, the characteristic $1^{\text {st }}$ order $\mathrm{DE}(\mathrm{P})$ peak is reached faster, i.e. after 86 seconds of irradiation, at which the SRG height is again $120 \mathrm{~nm}$ (Fig. 2b). There is also no significant out of plane component in the first order DE noticeable (see blue curve $1^{\text {st }}$ DE (S) in Fig. $2 b$ ).

With the above described set-up we are able to generate a reversible switching of the polymer topography between a structured and a flat state utilizing irradiation with changing interference patterns. This was achieved by changing the position of the mirror M4 (see Fig. 1) during irradiation which results in a lateral shift of the whole interference pattern along the polymer topography. Fig. 3 shows the results of shifting the interference pattern by half of the optical period for Pazo polymer film. The experiment is performed as follows: first the sample is irradiated for 180 seconds with RL polarization IP (onset of irradiation is shown by $1^{\text {st }}$ dashed white line at the top of Fig. 3a). Polarization IP means that the intensity along the sample is constant, while the orientation of the electric field vector (see white arrows in Fig. $2 \mathrm{c}$ and $3 \mathrm{a}$ ) varies locally with a certain period (optical period, 1 ). This first irradiation step generates a grating height of $40 \mathrm{~nm}$ (Fig. 3a). Afterwards the local distribution of the electrical field vector is shifted by half of the optical period, which corresponds to $90^{\circ}$ rotation of local electrical field vector (see white arrows in Fig. 3a). After the shift the SRG height continuously decreases till a flat topography is reached (dashed white line at the bottom of Fig. 3a). At this point the irradiation is stopped. This erase process is slightly faster $\left(160\right.$ s, i.e. $0.29 \mathrm{~nm} \mathrm{~s}^{-1}$ of topography flattening) compared to SRG inscription (180 s, $0.20 \mathrm{~nm} \mathrm{~s}^{-1}$ of topography increase). A possible explanation is that the initial state of both processes is different. During irradiation with IP and corresponding cyclic trans-cis photoisomerization the azobenzene groups rotate to align perpendicularly with their main axis to the electrical field vector resulting in locally ordered domains. Since in the case of the SRG formation the azobenzene molecules are initially randomly orientated within the polymer film, the time needed for local azobenzene alignment is larger than
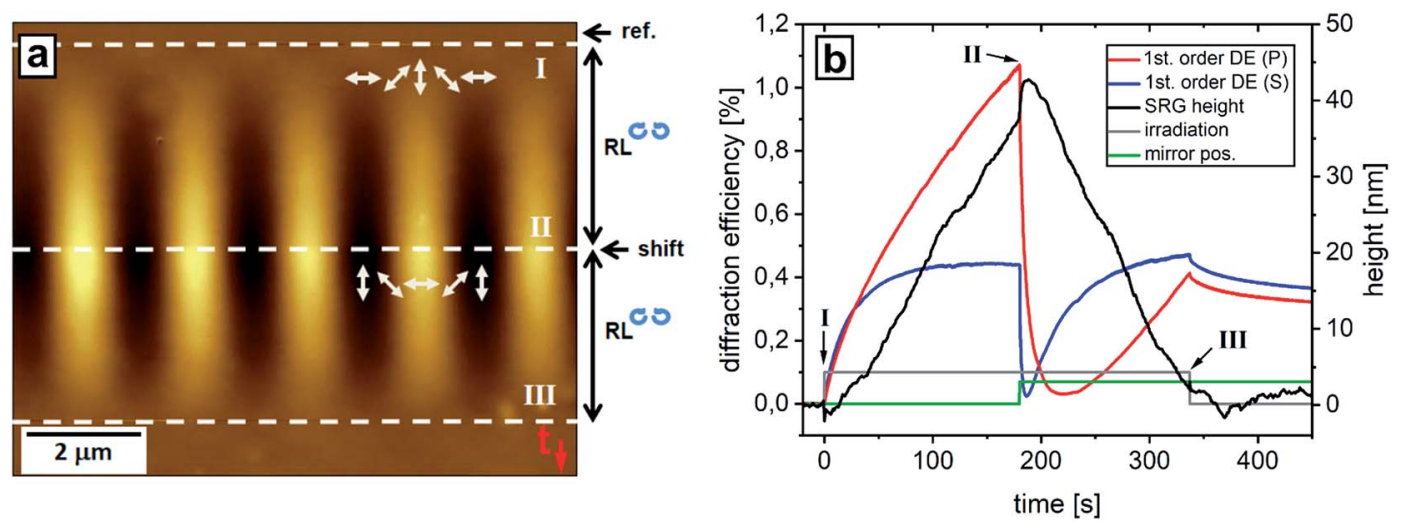

Fig. 3 (a) In situ recorded AFM micrograph of the Pazo polymer film during irradiation with $\mathrm{RL} I \mathrm{P}\left(\lambda=491 \mathrm{~nm}, I=200 \mathrm{~mW} \mathrm{~cm}^{-2} ; \Lambda=2 \mu \mathrm{m}\right)$, the local distribution of polarization is indicated by white arrows. AFM scanning starts at the top in dark with flat topography; at the point marked by the $1^{\text {st }}(\mathrm{I})$ white dashed line, the irradiation with IP is switched on for $180 \mathrm{~s}$; at the point marked by "shift" at the $2^{\text {nd }}$ (II) dashed white line, the interference pattern is shifted by half an optical period. The new distribution of the electrical field vector relative to the polymer topography is shown as above. The shifting of the interference pattern is equivalent to a rotation of the local electric field vectors by $90^{\circ}$. At the bottom of the micrograph marked by $3^{\text {rd }}$ (III) dashed white line, the irradiation is switched off. (b) In situ recorded SRG height and diffraction efficiency as a function of irradiation time. At the point where the interference pattern is shifted, the increase in the SRG height becomes noticeable (black curve), while the diffraction efficiency decreases (red and blue curves). The SRG is erased with time accompanied by the formation of a new birefringent grating in the bulk. 
in the case of irradiation with shifted interference patterns. In this case, due to local rotation of the electric field vector by $90^{\circ}$, the probability to absorb light by the aligned azobenzene molecules is larger thus resulting in a faster kinetics of topography response. At the point where the interference pattern is shifted, a fast jump in the SRG height of $5 \mathrm{~nm}$ became noticeable followed by a linear decrease (Fig. 3b, black curve). The explanation for this behavior is based again on local preorientation of the azobenzene molecules during the first step of irradiation. At the point of IP shifting all the aligned molecules are able to absorb incoming light resulting in noticeable increase of the free volume in polymer film and as a consequence its height increases.

The in-plane component of the diffraction efficiency of the first order diffraction (red curve, $1^{\text {st }}$ order DE (P), in Fig. 3b) continuously increases with SRG height. The out of plane component (blue curve, $1^{\text {st }}$ order DE (S), in Fig. 3b) saturates after a certain time. At the point where the interference pattern is shifted, there is fast drop (within 6 seconds) of out-of-plane component (blue curve in Fig. 3b), at this point the SRG height and the out-of-plane component contribute to the overall DE signal. After 37 seconds the in-plane component (red curve in Fig. 3b) settles to zero, while the increasing $1^{\text {st }}$ order DE (S) component and the left SRG result in DE signal of $0.4 \%$.

A short time after shifting of the IP, both components of the DE, i.e. in- and out-of-plane, start to increase, while the SRG height decreases. At the point where the topography becomes flat there is nevertheless still significant DE signal indicating the formation of a new birefringent grating from alignment of azobenzene groups in the bulk. In this case the erase process with shifting the interference pattern can be understood as erasing the primary, topographical SRG and forming a new birefringent grating in the bulk. Further irradiation will create a new SRG but shifted by half an optical period as described below (see Fig. 5).
A similar behavior is also observed in case of poly(MMA-coDR1A) polymer films (Fig. 4). Since the response of this polymer on irradiation is faster, the first exposure to RL IP lasts for only 48 seconds resulting in a grating height of $80 \mathrm{~nm}$ (speed of grating increase is $1.7 \mathrm{~nm} \mathrm{~s}^{-1}$ ). After shifting the interference pattern by half a period, the topography is flattened within only 34 seconds (speed of grating decrease is $2.1 \mathrm{~nm} \mathrm{~s}^{-1}$ ) (Fig. 4). At the point where the topography is flat again, there is a small signal in the $1^{\text {st }}$ order DE indicating the formation of new bulk phase grating. The out-of-plane component in the DE (blue curve, $1^{\text {st }}$ order DE (S), in Fig. $\left.4 \mathrm{~b}\right)$ is negligible. In contrast to the Pazo polymer film there is no jump in the SRG height detected at the point where the interference pattern is shifted. One possible explanation for this is that due to the fast response on irradiation, the topography jump is completed within a time too short for the AFM to detect it.

In the previous experiments the irradiation is stopped when the surface is flattened, but in case of further irradiation after IP shifting, the formation of a new SRG is observed with positions of maxima and minima interchanged (Fig. 5). Moreover, when the IP is shifted back to its initial distribution, the primary SRG is recovered. This procedure can be conducted repeatedly, as shown in Fig. 5, where the continuous scan with repeated shifts leads to a checkerboard pattern in the AFM recording. For the Pazo polymer, the irradiation is stopped after 4 cycles of IP shifting, at a point where the surface has just been rendered flat again (Fig. 5a).

The presence of the diffraction efficiency signal (red and blue curves in Fig. 5b) indicates the existence of bulk birefringent grating due to local alignment of the azobenzene side groups. This phase grating decreases in the dark indicating orientation relaxation of the azobenzenes with time. A similar experiment is performed in the case of poly(MMA-co-DR1A) polymer films that permits the same reversible surface structuring (Fig. 5c and d). In this case, the shifting is carried out seven times as indicated in Fig. 5c. Here, the irradiation time for each step is increased
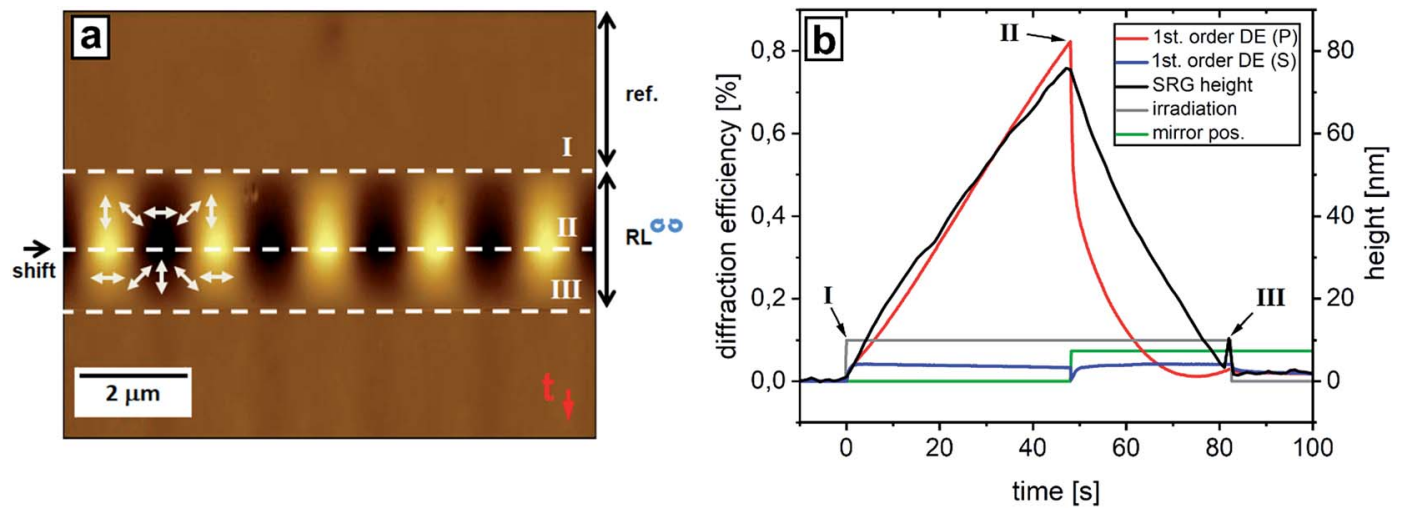

Fig. 4 (a) In situ recorded AFM micrograph of the SRG formation and erasure within the poly(MMA-co-DR1A) polymer film $\left(h_{\text {poly }}(M M A-c o-D R 1 A)=\right.$ $600 \mathrm{~nm})$ during irradiation with a RL interference pattern $\left(\lambda=491 \mathrm{~nm}, l=200 \mathrm{~mW} \mathrm{~cm}{ }^{-2} ; \Lambda=2 \mu \mathrm{m}\right)$. The AFM scanning proceeds from top to bottom (the direction is indicated by red arrow in the lower right corner), during scanning in dark, the topography is flat. At the position marked with the $1^{\text {st }}$ (I) dashed white line the irradiation with RL IP is switched on for 48 seconds. At the second dashed line (II) the interference pattern is shifted by $\lambda / 2$ (half of the optical period). The local distribution of the electric field vector relative to the topography extrema is shown by white arrows for both cases. After erasing of the polymer grating (34 seconds of irradiation), the laser is switched off (at the point marked by the $3^{\text {rd }}$ (III) dashed white line). (b) In situ recorded SRG height (black line) and $1^{\text {st }}$ order diffraction efficiency in plane (red line, $1^{\text {st }}$ order DE (P)) and out of plane (blue line, $1^{\text {st }}$ order DE (S)) as a function of time. 

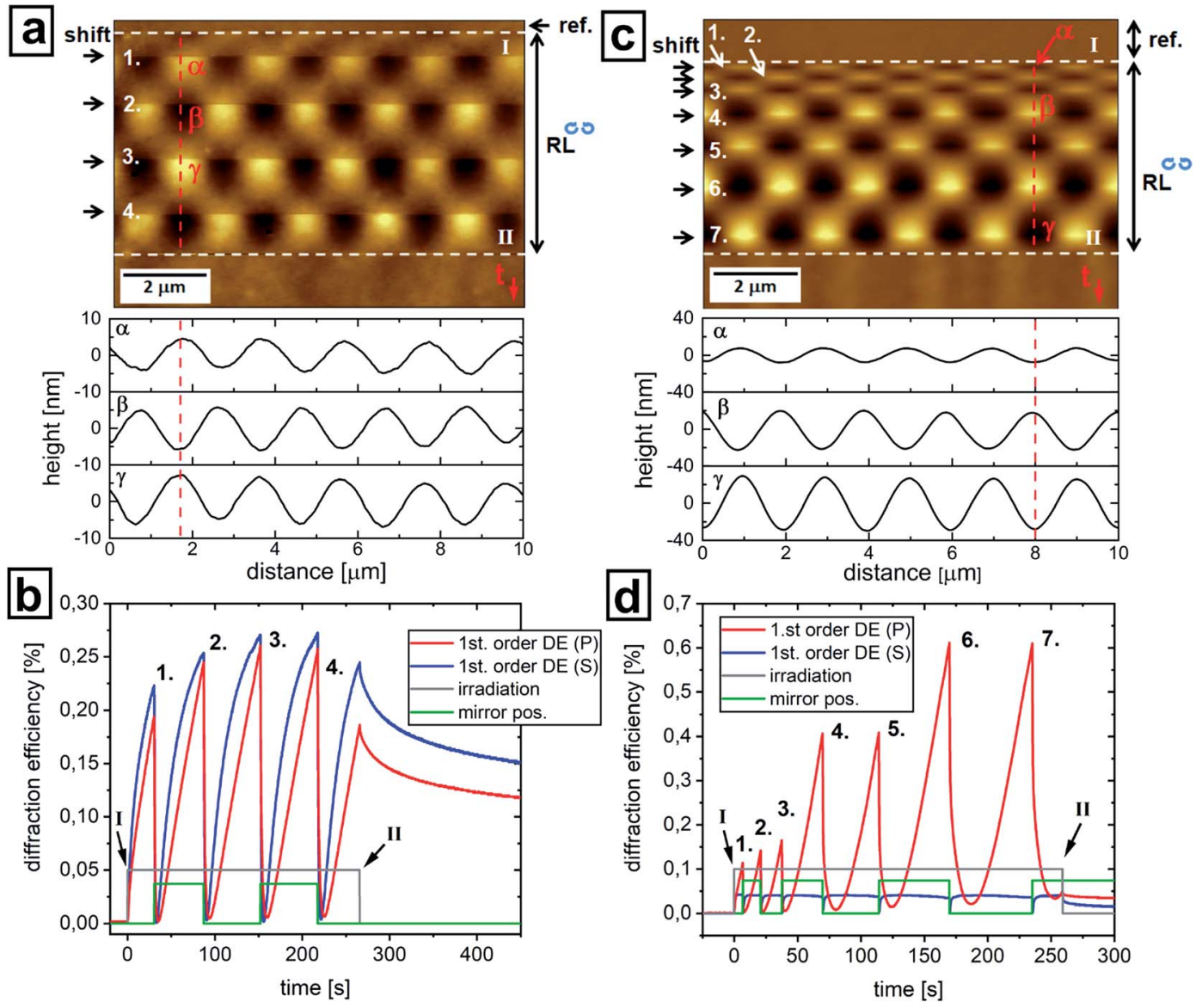

Fig. 5 (a) In situ recorded AFM micrograph of the Pazo polymer film $\left(h_{\text {Pazo }}=1 \mu \mathrm{m}\right)$ during irradiation with $\mathrm{RL} \mathrm{IP}\left(\lambda=491 \mathrm{~nm}, I=200 \mathrm{~mW} \mathrm{~cm}^{-2} ; \Lambda\right.$ $=2 \mu \mathrm{m})$. The interference pattern is shifted four times by $\lambda / 2$ of the optical period each after $60 \mathrm{~s}$ of irradiation. After each shifting of the IP the initial SRG is erased and a new SRG is formed with interchanged position of topography maxima and minima. The irradiation was stopped at the point where the surface was flat again, marked by dashed white line (II). (b) The evaluation of the $1^{\text {st }}$ order DE recorded in plane (red curve) and out-of-plane (blue curve) is presented as a function of time for four successive IP shifts. A drastic drop in the diffraction efficiency became noticeable whenever the interference pattern is shifted. (c) AFM micrograph of poly(MMA-CO-DR1A) film topography $\left(h_{\text {poly(MMA-co-DR1A })}=600\right.$ $\mathrm{nm}$ ) irradiated with periodically shifted RL IP with continuously increasing irradiation time: $7 \mathrm{~s}, 14 \mathrm{~s}, 17 \mathrm{~s}, 32 \mathrm{~s}, 44 \mathrm{~s}, 56 \mathrm{~s}, 65 \mathrm{~s}$. (d) The corresponding $1^{\text {st }}$ order DE recorded simultaneously with the topography change in (c). Red line in (a) and (c) indicates the direction along which the cross-section was recorded as indicated in the corresponding plots below (a) and (c).

between successive shifting events resulting in a continuous increase in SRG height showing also here the independency of the erase process from the SRG height. By continuously shifting the interference pattern, e.g. by applying a saw-tooth signal to the piezo, one can shift the SRG over several micrometers (Fig. 6).

In this way, we are able to induce fluctuations in the polymer topography resembling propagation of the water wave (Fig. 6b). To achieve continuous change in the polymer topography without intermittent flat state, we shift the IP in each subsequent step by fourth of the optical period which results in propagation of the primary topography maxima/minima over 14 $\mu \mathrm{m}$ to the right (see Fig. $6 \mathrm{~b}$ ). The corresponding changes in the DE signal are shown in Fig. S2 (see ESI $\dagger$ ).

Summarizing this part we can state that the erasure of the polymer topography by applying shifts to the interference patterns (i.e. rotating the local distribution of the electric field vector by $90^{\circ}$ ) is an efficient and fast process which proceeds by overwriting the initial SRG by a new bulk birefringent grating. In order to estimate the efficiency of this erase mechanism, we compare in the following how erasure can be achieved alternatively by applying a single beam or by thermal treatment.

\section{Erasing a SRG pattern with a single laser beam}

A fast optical switch is integrated into the set-up in order to turn off one of the two interfering beams. This is achieved by employing a Pockels cell and an additional polarizer (see Fig. 1). By applying the lambda-half voltage on the Pockels cell the polarization of the writing beam is rotated by $90^{\circ}$ so that the light will be blocked by the subsequent polarizer. This established a very fast switching between two-beam and single beam erasure. Due to the two integrated lambda quarter plates of the RL interference pattern, the single beam is also circularly polarized, which can be expected to induce an effective erasure process. ${ }^{48}$ Fig. 7 shows the results of the single beam experiment performed with a poly(MMA-co-DR1A) structured polymer film. 


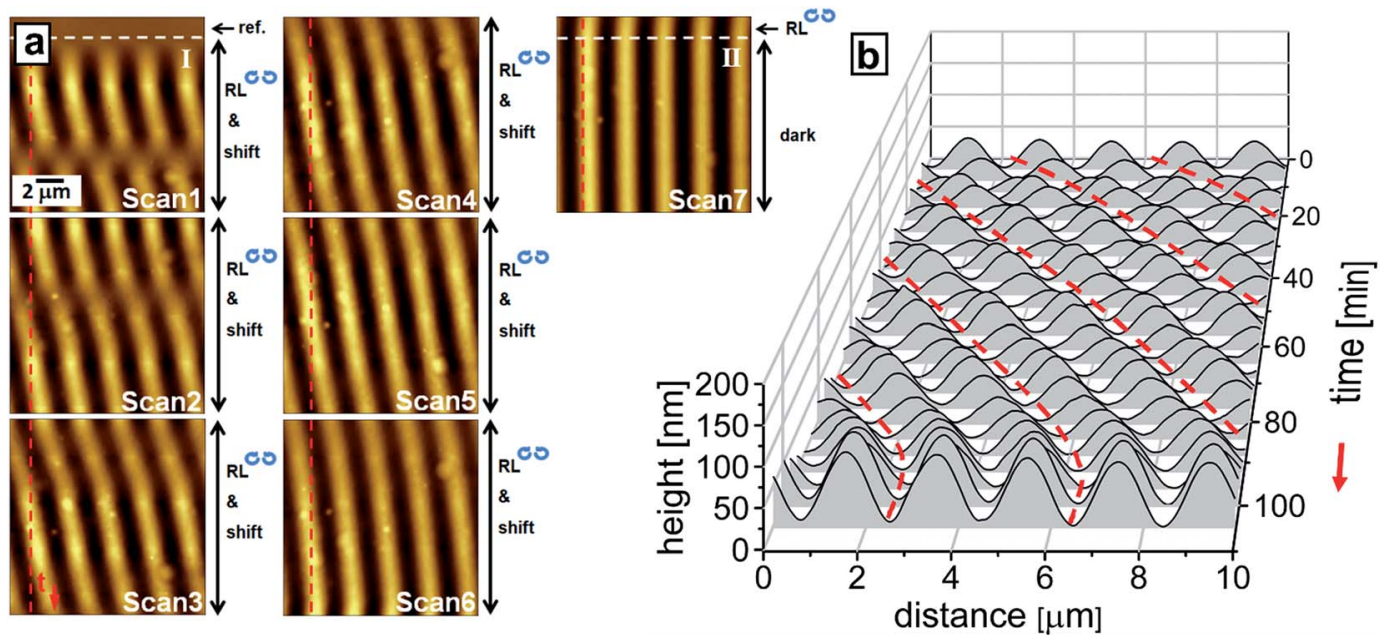

Fig. 6 (a) AFM micrographs of the Pazo polymer irradiated by shifting the RL IP $26 \times$ times by $\lambda / 4$, which corresponds with a total shift of ca. 7 periods of the optical grating, i.e. over $14 \mu \mathrm{m}$ distance. The dashed red vertical line indicates the position of one of the primary topography maxima. With the IP shifting the SRG propagates to the right resembling a traveling wave schematically shown in (b)

The film was first irradiated with RL IP for 1 minute resulting in $80 \mathrm{~nm}$ SRG height. Afterwards the writing beam is switched off for 10 minutes. During this time the in-plane component of the $1^{\text {st }}$ order DE drops exponentially while the SRG height stays constant (Fig. 7). In the following step switching on the erase beam results in fast decrease of the DE signal and SRG height. The irradiation is stopped after $400 \mathrm{~min}$ when no diffraction efficiency signal could be detected. At this point the polymer film is found to be atomically smooth (see Fig. 7c). In the case of Pazo polymer the erasure with the circularly polarized beam does not result in a complete flattening of the polymer topography as well as vanishing of DE signal even after $20 \mathrm{~h}$ of irradiation (see ESI, Fig. S1†). Comparing these results with those obtained by the shifting procedure (Fig. 3 and 4 ) shows that erasure via shifting is indeed much faster for both polymers, but the final state of the flattened polymer film is different. In the case of single beam erasure, the alignment of azobenzene molecules introduced by the IP irradiation is getting destroyed and transformed to a random orientation indicated by decreasing of DE signal. Shifting the interference pattern eliminates the grating topography by overwriting the initial birefringent grating with a new one. At the point of a flat surface the bulk is already reoriented with a pure phase grating which can directly be seen in the DE (red and blue curves in Fig. 3b).

To compare the optically induced SRG erasure process to the thermal one, we employed another home-made set-up consisting of a hot plate with a temperature sensor onto which the sample is placed, and an optical part facilitating red laser beam
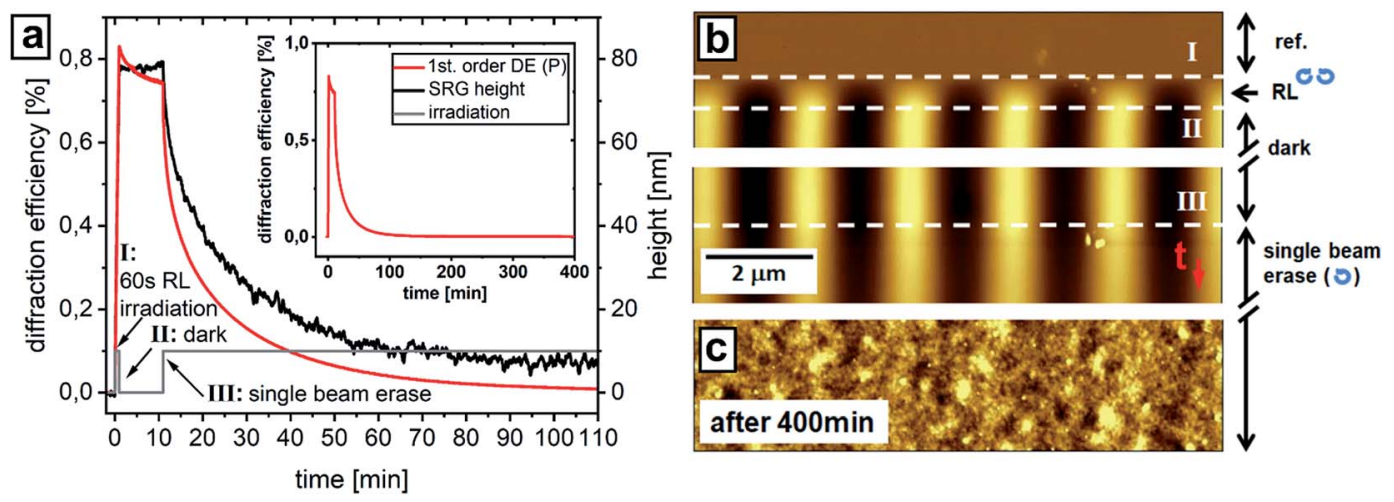

Fig. 7 Single beam erasure experiment of the SRG in a poly(MMA-co-DR1A) film. (a) In situ recorded SRG height (black curve) and in plane component of the $1^{\text {st }}$ order DE signal (red curve) as a function of time. The gray curve indicates irradiation steps. The polymer film is illuminated with RL interference pattern for 1 min resulting in $80 \mathrm{~nm}$ SRG height (see AFM topography in "b"). Afterwards the irradiation is switched off for $10 \mathrm{~min}$. The $1^{\text {st }}$ order DE signal decreases with time, while the SRG height is constant. In the following step, the circularly polarized erase beam is switched on resulting in a decay of the SRG height and DE signal. (b) In situ recorded AFM micrograph showing the change in the SRG height as a function of time. The scanning is started at the top in dark where the topography is flat followed by 1 min irradiation with RL IP started at point marked by $1^{\text {st }}(\mathrm{I})$ and stopped at $2^{\text {nd }}$ (II) dashed white line, after 10 minutes scanning in dark, the circular polarized erasure beam is switched on ( ${ }^{\text {rd }}$ white dashed line (III)). (c) Polymer film surface after $400 \mathrm{~min}$ of single beam irradiation. The SRG (80 nm) is completely erased and the DE reached its initial value of zero $\left(I_{\mathrm{RL}}=200 \mathrm{~mW} \mathrm{~cm}{ }^{2} ; I_{\text {single beam }}=100 \mathrm{~mW} \mathrm{~cm}^{2} ; \Lambda=2 \mu \mathrm{m} ; h_{\text {poly(MMA-co-DR1A) }}=600 \mathrm{~nm}\right)$. 

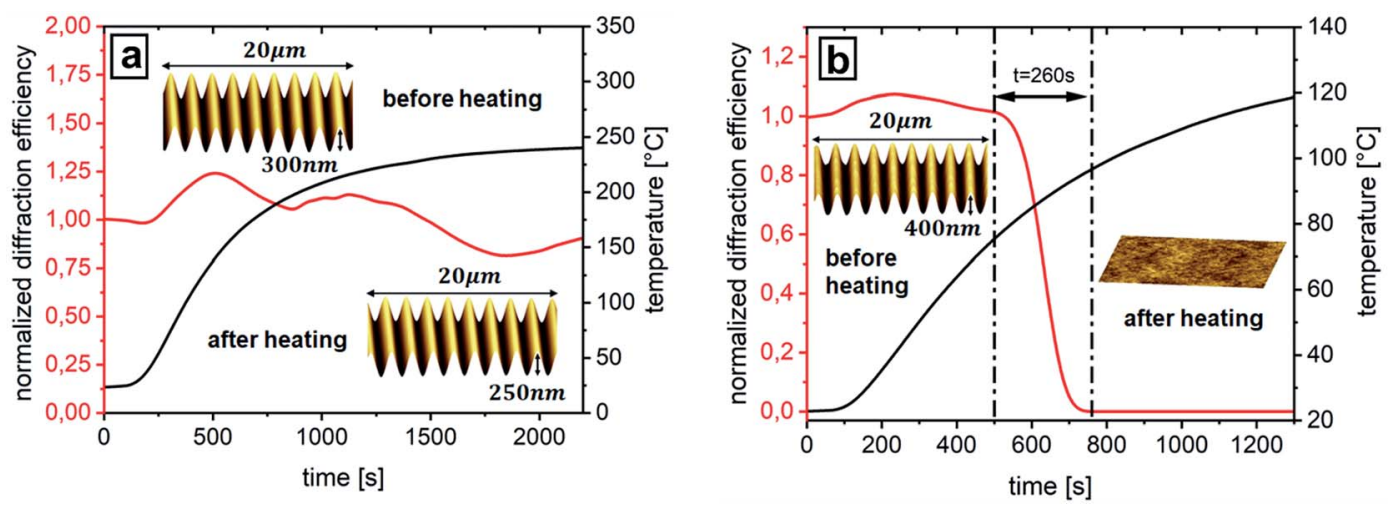

Fig. 8 Thermal treatment of the surface relief grating: diffraction efficiency (red curves) of the Pazo (a) and poly(MMA-co-DR1A) film (b) as a function of time during temperature increase. The Pazo film shows no significant drop in the DE as well as in grating height under heating up to $250^{\circ} \mathrm{C}$. The poly(MMA-co-DR1A) film shows a fast drop in the DE down to zero within 260 seconds initiated at $80^{\circ} \mathrm{C}$. The ex situ acquired AFM micrographs show that the $400 \mathrm{~nm}$ SRG was completely erased $\left(I=200 \mathrm{~mW} \mathrm{~cm}{ }^{2} ; \Lambda=2 \mu \mathrm{m} ; h_{\text {poly(MMA-co-DR1A })}=600 \mathrm{~nm} ; h_{\text {Pazo }}=1 \mu \mathrm{m}\right)$.

for measurements of diffraction efficiency to be directed at the polymer sample. As can be seen from Fig. 8 the erasure of a $400 \mathrm{~nm}$ high grating of poly(MMA-co-DR1A) sets in at $c a .80^{\circ} \mathrm{C}$ $\left(T_{\mathrm{g}} \sim 102{ }^{\circ} \mathrm{C}\right)^{58}$ and after $\mathrm{ca} .260$ seconds the diffraction efficiency signal drops to zero (Fig. 8b). So, the thermal erasure needs around 5 minutes and heating up to $100^{\circ} \mathrm{C}$, while optical erasure by IP shifting takes place within 34 seconds at room temperature. Single beam erasure requires the most time $(400$ min). In contrast, it was not possible to flatten Pazo film thermally. Indeed, heating up to $250^{\circ} \mathrm{C}$, at which the decomposition of the polymer materials sets in (see TGA measurement in ESI, Fig. S3a $\dagger$ ), does not result in decrease of the diffraction efficiency signal (red curve in Fig. 8a), the SRG height is also only slightly affected: it drops from 300 to $250 \mathrm{~nm}$ within 30 minutes of heating at $250{ }^{\circ} \mathrm{C}$. As can be seen from DSC measurements (ESI, Fig. S3b†), there is no clear indication of the glass transition temperature. This result is in contrast to the work published by Ferreira et al., who measured $95{ }^{\circ} \mathrm{C}$ as $T_{\mathrm{g}}$ for this polymer, ${ }^{59}$ but in a good agreement with the results of Stumpe et $a .^{60}$ where it was reported that the SRG inscribed in Pazo could not be erased by heating at $200^{\circ}$ over 6 hours. This is explained by the presence of ionic interactions in the side chains of the Pazo (see chemical structure in Fig. 2a). ${ }^{61,62}$ Thus with these results, we can state that the erasure of the surface relief grating of Pazo polymer can be done quite easily and fast (160 seconds of irradiation at room temperature) using the IP shifting method (Fig. 3), with single beam the procedure takes 20 hours and does not result in complete elimination of the grating, while the thermal erasure is not possible at all. These experiments show that the temperature stability of the SRG depends on the polymer structure itself.

\section{Conclusions}

Here we have reported on the reversible surface structuring of photosensitive polymer films using a novel method of fast SRG erasure simply by shifting the interference pattern by half an optical period. This is achieved by utilizing a home-made set-up to record the changes in the surface relief grating (SRG) and the diffraction efficiency (DE) signal upon irradiation with varying interference patterns in situ. Our setup also permits to separate the SRG part from the birefringent grating component in the $\mathrm{DE}$, so that we can directly probe the molecular orientation of the azobenzene moieties in the bulk. Integrating a phase delay stage into the two beam interference set-up allows for shifting the interference patterns along the sample plane; in this way the polymer surface can be rendered "dynamic". A shift of half of the optical period leads to erasure of the just created surface grating by inscribing a new birefringent grating in the bulk. Depending on the polymer material this leads to the generation of a pure phase grating without any surface structure (with the interesting example of the Pazo polymer studied here). The reversibility of the surface structuring is demonstrated for two different polymers. Attempts to erase the SRG by single beam irradiation and a change in the temperature required significantly more effort than optical shifting method. In the case of thermal erasure, the Pazo polymer is found to be stable such that both gratings, topographical and the bulk birefringence withstand temperatures up to $250{ }^{\circ} \mathrm{C}$ over hours of heating, while in the poly(MMA-co-DR1A) film the SRG is erased completely with heating at the glass transition temperature $T_{\mathrm{g}}$ during 5 minutes. Erasing SRGs with a single circularly polarized beam is feasible, but requires 400 minutes of irradiation time for poly(MMA-co-DR1A), while the more rigid polymer Pazo takes more than 20 hours of irradiation. In contrast, the optical shift method reduces these times to only 160 seconds and 34 seconds irradiation time for Pazo and poly(MMA-co-DR1A) polymers, respectively. This phenomenon is simply related to the fact that shifting the interference pattern produces a pure phase grating with a flat topography, while erasing with a single beam completely destroy the grating restoring the initial amorphous state of the polymer film and thus requires more time. The method proposed here bears one decisive advantage: patterns in topography and other optical properties such as birefringence are inscribed at the same time. By using the peculiar dynamics and depending on the protocol of optical 
settings we can end up with topographical and optical (birefringence) patterns, or combinations thereof. This might lead to hitherto unprecedented applications as indicated in the introduction.

\section{Conflicts of interest}

There are no conflicts to declare.

\section{Acknowledgements}

This research is supported by the Helmholtz Graduate School on Macromolecular Bioscience (Teltow, Germany) and GermanRussian Interdisciplinary Science Center (G-RISC). We thank Prof. Dr Carsten Henkel and Burkhard Stiller for the fruitful discussions. We thank Prof. I. Zvereva (Centre of Thermal Analysis and Calorimetry, St. Petersburg State University) for the support in measurements and interpretation of TGA and DSC results.

\section{References}

1 D. Liu and D. J. Broer, Responsive Polymer Surfaces: Dynamics in Surface Topography, Wiley-VCH, Weinheim, Germany 2017.

2 L. Dong, A. K. Agarwal, D. J. Beebe and H. Jiang, Nature, 2006, 442, 551.

3 T. M. de Jong, D. K. G. de Boer and C. W. M. Bastiaansen, Opt. Express, 2011, 19, 15127-15142.

4 M. Yamada, M. Kondo, J. Mamiya, Y. Yu, M. Kinoshita, C. J. Barrett and T. Ikeda, Angew. Chem., Int. Ed. Engl., 2008, 47, 4986.

5 S. Hvilsted, C. Sanchez and R. Alcala, J. Mater. Chem., 2009, 19, 6641-6648.

6 M. Ibn-Elhaj and M. Schadt, Nature, 2001, 410, 796.

7 T. S. Wong, S. H. Kang, S. K. Y. Tang, E. J. Smythe, B. D. Hatton, A. Grinthal and J. Aizenberg, Nature, 2011, 477, 443.

8 L. M. Goldenberg, V. Lisinetskii, Y. Gritsai, J. Stumpe and S. Schrader, Laser Phys. Lett., 2013, 10, 085804.

9 L. M. Goldenberg, V. Lisinetskii, Y. Gritsai, J. Stumpe and S. Schrader, Adv. Mater., 2012, 24, 3339.

10 T. Alasaarela, D. Zheng, L. Huang, A. Priimagi, B. Bai, A. Tervonen, S. Honkanen, M. Kuittinen and J. Turunen, Opt. Lett., 2011, 36, 2411-2413.

11 R. H. Bishop, in Mechatronics: An Introduction, ed. T. Francis, Taylor and Francis Group, Oxfordshire, England, 2006, p. 312.

12 S. Loebner, J. Jelken, N. S. Yadavalli, E. Sava, N. Hurduc and S. Santer, Molecules, 2016, 21, 1663.

13 S. L. Oscurato, F. Borbone, P. Maddalena and A. Ambrosio, ACS Appl. Mater. Interfaces, 2017, 9, 30133-30142.

14 N. A. Malvadkar, M. J. Hancock, K. Sekeroglu, W. J. Dressick and M. C. Demirel, Nat. Mater., 2010, 9, 1023.

15 P. Zhang, H. Liu, J. Meng, G. Yang, X. Liu, S. Wang and L. Jiang, Adv. Mater., 2014, 26, 3131-3135.

16 X. Qiu, J. Appl. Phys., 2010, 108, 011101.
17 D. Liu, N. B. Tito and D. J. Broer, Nat. Commun., 2017, 8, 1526.

18 W. Feng, D. J. Broer and D. Liu, Adv. Mater., 2018, 30, 1704970.

19 A. H. Gelebart, D. J. Mulder, M. Varga, A. Konya, G. Vantomme, E. W. Meijer, R. L. B. Selinger and D. J. Broer, Nature, 2017, 546, 632-636.

20 K. Kumar, A. P. H. J. Schenning, D. J. Broer and D. Liu, Soft Matter, 2016, 12, 3196-3201.

21 A. J. J. Kragt, D. J. Broer and A. P. H. J. Schenning, Adv. Funct. Mater., 2018, 28, 1704756.

22 D. Liu, L. Liu, P. R. Onck and D. J. Broer, Proc. Natl. Acad. Sci. U. S. A., 2015, 112, 3880-3885.

23 A. Kopyshev, C. J. Galvin, R. R. Patil, J. Genzer, N. Lomadze, D. Feldmann, J. Zakrevski and S. Santer, ACS Appl. Mater. Interfaces, 2016, 8, 19175.

24 P. Rochon, E. Batalla and A. Natansohn, Appl. Phys. Lett., 1995, 66, 136-138.

25 D. Y. Kim, S. K. Tripathy, L. Li and J. Kumar, Appl. Phys. Lett., 1995, 66, 1166-1168.

26 C. J. Barrett, P. Rochon and A. Natansohn, J. Chem. Phys., 1998, 109, 1505-1516.

27 K. G. Yager and C. J. Barrett, Curr. Opin. Solid State Mater. Sci., 2001, 5, 487-494.

28 T. König, N. S. Yadavalli and S. Santer, J. Mater. Chem., 2012, 22, 5945-5950.

29 T. König, V. V. Tsukruk and S. Santer, ACS Appl. Mater. Interfaces, 2013, 5, 6009-6016.

30 Th. Papke, N. S. Yadavalli, C. Henkel and S. Santer, ACS Appl. Mater. Interfaces, 2014, 6, 14174-14180.

31 A. Kopyshev, C. J. Galvin, J. Genzer, N. Lomadze and S. Santer, Polymer, 2016, 98, 421-428.

32 A. Kopyshev, N. Lomadze, D. Feldmann, J. Genzer and S. Santer, Polymer, 2015, 79, 65-72.

33 J. Vapaavuori, A. Goulet-Hanssens, I. T. S. Heikkinen, C. J. Barrett and A. Priimagi, Chem. Mater., 2014, 26, 50895096.

34 J. E. Koskela, J. Vapaavuori, R. H. A. Ras and A. Priimagi, ACS Macro Lett., 2014, 3, 1196-1200.

35 N. S. Yadavalli, T. König and S. Santer, J. Soc. Inf. Disp., 2015, 23, 154-162.

36 T. König, L. M. Goldenberg, O. Kulikovska, L. Kulikovsky, J. Stumpe and S. Santer, Soft Matter, 2011, 7, 4174-4178.

37 V. Toshchevikov, J. Ilnytskyi and M. Saphiannikova, J. Phys. Chem. Lett., 2017, 8, 1094-1098.

38 N. S. Yadavalli, D. Korolkov, J. Moulin, M. Krutyeva and S. Santer, ACS Appl. Mater. Interfaces, 2014, 6, 11333-11340.

39 N. S. Yadavalli, F. Linde, A. Kopyshev and S. Santer, ACS Appl. Mater. Interfaces, 2013, 5, 7743-7747.

40 G. Di Florio, E. Bründermann, N. S. Yadavalli, S. Santer and M. Havenith, Nano Lett., 2014, 14, 5754-5760.

41 J. Ilnytskyi and M. Saphiannikova, ChemPhysChem, 2015, 16, 3180-3189.

42 J. M. Ilnytskyi, D. Neher and M. Saphiannikova, J. Chem. Phys., 2011, 135, 044901.

43 V. Toshchevikov, M. Saphiannikova and G. Heinrich, J. Phys. Chem. B, 2012, 116, 913-924. 
44 V. Toshchevikov and M. Saphiannikova, J. Phys. Chem. B, 2014, 118, 12297-12309.

45 V. Toshchevikov, M. Saphiannikova and G. Heinrich, J. Phys. Chem. B, 2009, 113, 5032-5045.

46 K. G. Yager and C. J. Barrett, J. Chem. Phys., 2007, 126, 094908.

47 T. M. Geue, M. G. Saphiannikova, O. Henneberg, U. Pietsch, P. L. Rochon and A. L. Natansohn, Phys. Rev. E: Stat., Nonlinear, Soft Matter Phys., 2002, 65, 052801.

48 J. Vapaavuori, R. H. A. Ras, M. Kaivola, C. G. Bazuin and A. Priimagi, J. Mater. Chem. C, 2015, 3, 11011-11016.

49 A. Tofini, L. Levesque, O. Lebel and R. G. Sabat, J. Mater. Chem. C, 2018, 6, 1083-1091.

50 F. L. Labarthet, T. Buffeteau and C. Sourisseau, J. Phys. Chem. B, 1998, 102, 2654-2662.

51 F. L. Labarthet, P. Rochon and A. Natansohn, Appl. Phys. Lett., 1999, 75, 1377-1379.

52 F. L. Labarthet, J. L. Bruneel, T. Buffeteau and C. Sourisseau, J. Phys. Chem. B, 2004, 108, 6949-6960.
53 N. S. Yadavalli and S. Santer, J. Appl. Phys., 2013, 113, 224304. 54 N. S. Yadavalli, M. Saphiannikova, N. Lomadze, L. M. Goldenberg and S. Santer, Appl. Phys. A, 2013, 113, 263-272.

55 N. S. Yadavalli, M. Saphiannikova and S. Santer, Appl. Phys. Lett., 2014, 105, 051601.

56 A. Emoto, S. B. Baharim, T. Sasaki, T. Shioda, A. Ogiwara and H. Ono, Jpn. J. Appl. Phys., 2010, 49, 122502.

57 C. V. Raman and N. S. Nagendra Nathe, Proc. Indian Acad. Sci. Sect. A, 1935, 2, 406-412.

58 As provided by Sigma Aldrich.

59 Q. Ferreira, P. A. Ribeiro, O. N. Oliveira and M. Raposo, ACS Appl. Mater. Interfaces, 2012, 4, 1470-1477.

60 L. M. Goldenberg, O. Kulikovska and J. Stumpe, Langmuir, 2005, 21, 4794.

61 Q. Zhang, X. Wang, C. J. Barrett and C. G. Bazuin, Chem. Mater., 2009, 21, 3216.

62 J. Vapaavuori, C. G. Bazuin and A. Priimagi, J. Mater. Chem. C, 2018, 6, 2168. 